\title{
Mapping QTLs for nitrogen uptake in relation to the early growth of wheat (Triticum aestivum L.)
}

Diaoguo $\mathrm{An}^{1,2,3, \dagger}$, Junying $\mathrm{Su}^{1,3, \dagger}$, Quanyou $\mathrm{Liu}^{2}$, Yongguan $\mathrm{Zhu}^{2}$, Yiping Tong ${ }^{1,5}$, Junming $\mathrm{Li}^{1}$, Ruilian Jing ${ }^{4}$, Bin $\mathrm{Li}^{1} \&$ Zhensheng $\mathrm{Li}^{1}$

${ }^{1}$ The State Key Laboratory of Plant Cell and Chromosome Engineering, Institute of Genetics and Developmental Biology, Chinese Academy of Sciences, 100101, Beijing, China. ${ }^{2}$ Research Center for Eco-environmental Sciences, Chinese Academy of Sciences, 100085, Beijing, China. ${ }^{3}$ Graduated School, Chinese Academy of Sciences, 100039, Beijing, China. ${ }^{4}$ Institute of Crop Science, Chinese Academy of Agricultural Sciences, 100081, Beijing, China. ${ }^{5}$ Corresponding author*

Received 29 November 2005. Accepted in revised form 5 February 2006

Key words: early vigor, nitrogen uptake, nitrogen use efficiency, QTLs, Triticum aestivum L.

\begin{abstract}
The objective of this study was to map QTLs for N uptake (NUP) in wheat, and to investigate factors influencing NUP. Two independent field trials with low $\mathrm{N}(\mathrm{LN})$ and high $\mathrm{N}(\mathrm{HN})$ treatments were conducted in the growing seasons of 2002-2003 (trial 1) and 2003-2004 (trial 2) to measure NUP per plant (N accumulated in the aerial part at maturity stage) of a doubled haploid (DH) population consisting of 120 DH lines derived from winter wheat varieties Hanxuan 10 and Lumai 14. A hydroponic culture with all nutrients supplied sufficiently was conducted to investigate shoot dry weight (SDW), root dry weight (RDW), tiller number (TN) and NUP (total plant $\mathrm{N}$ uptake) per plant of this mapping population at seedling stage. SDW, RDW, TN and NUP investigated in the hydroponic culture were significantly and positively correlated with each other, and with NUP under both LN and HN conditions in the field trials. Nine and eight QTLs for NUP were detected under LN and HN conditions in the field trials, respectively. Four to five QTLs for SDW, RDW, TN and NUP were detected in the hydroponic culture. One SDW QTL, three RDW QTLs, two TN QTLs detected in the hydroponic culture were linked with QTLs for NUP under LN or HN condition in the field trials. The positive correlation and genetic linkage for the traits between the field trials and the hydroponic culture demonstrated that greater seedling vigor of root and shoot is an important factor influencing $\mathrm{N}$ uptake in wheat.
\end{abstract}

\section{Introduction}

Nitrogen $(\mathrm{N})$ fertilizer is required to maximize crop yields in many agricultural systems. To meet the food requirement of the increasing population, $\mathrm{N}$ fertilizer consumption in China increased rapidly in the past decades, and became the highest in the world in 1985. In 2000, China used more than $24 \mathrm{Mt}$ of pure $\mathrm{N}$, or about $30 \%$

\footnotetext{
${ }^{\dagger}$ These authors contributed equally to this work.

*FAX No: 86-10-64873482.

E-mail: yptong@genetics.ac.cn
}

of the world's total consumption (Ju et al., 2004, and references therein). Of the applied fertilizer $\mathrm{N}$ in Chinese agricultural systems, only about $30-35 \%$ is recovered by the crops, as high as $45-$ $50 \%$ losses and enters the environment (Zhu and Chen, 2002). Low recovery rate and high loss of fertilizer $\mathrm{N}$ would increase the cost of food production and the eutrophication of many natural aquatic and terrestrial ecosystems and contribute to atmospheric accumulation of greenhouse gases (Tilman, 1999). To increase $\mathrm{N}$ recovery rate and reduce $\mathrm{N}$ loss, systematic approaches are required, including optimizing management 
practices and breeding crops with improved $\mathrm{N}$ use efficiency (NUE) (Raun and Johnson, 1999).

Wheat (Triticum aestivum L.) is cultivated globally as well as in China; therefore breeding wheat with improved NUE is important in the development of sustainable agricultural systems that are more economically efficient and environmentally friendly. Genetic variation for NUE in wheat both under low (LN) and high (HN) N levels has been reported by many (Dhugga and Waines, 1989; Ortiz-Monasterio et al., 1997; Van Sanford and Mackown, 1986; Van Sanford et al., 1991), indicating the feasibility of improving wheat NUE genetically. It would be desirable to combine the breeding goals of yield improvement for conditions with high input of $\mathrm{N}$ fertilizers and yield improvement for low $\mathrm{N}$ input conditions (Presterl et al., 2003). Understanding the genetic control of NUE and related traits in wheat under both LN and HN conditions would help to achieve these breeding goals.

Physiological and molecular studies on $\mathrm{N}$ uptake and assimilation pathways reveal the highly complex of $\mathrm{N}$ use in plant (for review see Crawford and Forde, 2002). Quantitative trait locus (QTL) analysis based on high-density molecular linkage maps makes it possible to understand the genetic basis underlying this complex trait. QTLs regulating $\mathrm{N}$ use have been successfully studied in rice (Ishimaru et al., 2001; Lian et al., 2005; Obara et al., 2001, 2004; Yamaya et al., 2002), maize (Agrama et al., 1999; Bertin and Gallais, 2000; Gallais and Hirel, 2004; Hirel et al., 2001), and Arabidopsis (Loudet et al., 2003) under low $\mathrm{N}$ stress and normal $\mathrm{N}$ conditions. In wheat, major QTLs for grain yield components (ears per plant, grains per ear, and thousands grain weight) under low $\mathrm{N}$ stress were mapped on chromosomes 4AS, 7AL, 7BL, and around centromeres of chromosomes $4 \mathrm{~B}$ and $6 \mathrm{~A}$ using a spring wheat doubled haploid $(\mathrm{DH})$ population derived from the cross Chinese Spring $\times$ SQ1 (Quarrie et al., 2005). However, the genetic control of the two NUE components $\mathrm{N}$ uptake (NUP) and utilization efficiency (UTE, biomass or grain yield produced by unit $\mathrm{N}$ taken up) in wheat is still not well understood. Several lines of evidence show that NUP is more closely correlated with grain yield than UTE in wheat (Dhugga and Waines, 1989; Van Sanford and Mackown, 1986). Therefore, it can be assumed that improving NUP would be more efficient in the improvement of grain yield than improving UTE. Furthermore, improving NUP would reduce the amount of $\mathrm{N}$ entering the environment. The objective of this study was therefore to detect QTLs governing NUP and factors affecting NUP in wheat. In the following sections the results of our study were described, together with discussions on genetic control of NUP in relation to the early development of wheat plant.

\section{Materials and methods}

\section{Plant materials}

One hundred and twenty double haploid lines (DHLs) derived from winter wheat varieties Hanxuan 10 and Lumai 14, and their parents were used in all experiments. The DH population was constructed using anther culture of F1 plants (Jing et al., 1999). The parents were initially chosen for their difference in drought tolerance. Female parent Hanxuan 10, a drought-tolerate winter wheat variety released in 1966, was widely grown under rain-fed condition in semi-arid areas; while male parent Lumai 14, a high grain yield winter wheat variety released in 1986, was widely cultivated under irrigated condition (Jing et al., 1999; Hao et al., 2003). Of the original 221 DHLs, 150 lines were randomly selected to construct genetic map (Hao et al., 2003). In present study, 120 lines were selected at random from these 150 lines to evaluate the phenotypes.

\section{Evaluation of phenotypes}

\section{Field trials}

Two field trials were conducted in the growing seasons of 2002-2003 (trial 1) and 2003-2004 (trial 2) in the experimental station of Institute of Genetics and Developmental Biology, Chinese Academy of Sciences in Beijing. The average nitrate concentration in $0-75 \mathrm{~cm}$ soil profile sampled before fertilization treatment was $15 \mathrm{mg} \mathrm{N} \mathrm{kg}^{-1}$ soil in 2002 and $6 \mathrm{mg} \mathrm{N} \mathrm{kg}^{-1}$ in 2003. In each trial two $\mathrm{N}$ treatments were used, in low $\mathrm{N}(\mathrm{LN})$ treatment nil $\mathrm{N}$ was applied in trial 1 , and $30 \mathrm{~kg} \mathrm{hm}^{-2}$ of $\mathrm{N}$ (urea) was applied at stem elongation stage in trial 2; in high $\mathrm{N}(\mathrm{HN})$ treatment $180 \mathrm{~kg} \mathrm{hm}^{-2}$ of $\mathrm{N}$ was applied in both 
trials with 120 before sowing and 60 at stem elongation stage. Fifty-nine $\mathrm{kg} \mathrm{hm}^{-2}$ of $\mathrm{P}$ and $74 \mathrm{~kg} \mathrm{hm}^{-2}$ of $\mathrm{K}$ were applied as potassium dihydrophosphate before sowing in both treatments in both trials. A random block design with four replications was used in both trials. Seeds were sown at the end of September, and plants were harvested in the middle of next June at physiological maturity. For each DHL, 50 seeds were sown in a $1.5-\mathrm{m}-$ long row, and rows were spaced $23-\mathrm{cm}$ apart. DHLs were grouped into two according to plant height; DHL groups in each plot and DHLs in each group were randomly positioned.

Six wheat plants were sampled in each row at flowering to investigate aerial plant biomass (data not reported in the present paper), and the remaining plants (typically $30-35$ plants per row) were harvested at physiological maturity. Biological (dry weight of above-ground plant part) and grain yields were recorded on representative plants. Total $\mathrm{N}$ concentration in straw (aboveground plant part with grain removed) and grain tissues were measured using a semiautomated Kjeldahl method (Tecator Kjeltec Auto 1030 Analyzer, Tecator, Hogänäs, Sweden). NUP (total $\mathrm{N}$ accumulated in the above ground parts per plant) was calculated as the sum of $\mathrm{N}$ accumulated in straw (straw dry weight $\times$ straw $\mathrm{N}$ concentration) and in grain (grain yield $\times$ grain $\mathrm{N}$ concentration).

\section{Hydroponic culture}

To analyze the effects of early growth of wheat plant on $\mathrm{N}$ uptake, a hydroponic culture was carried out from March 25 to April 28 in 2004. Seeds were germinated at $25^{\circ} \mathrm{C}$ for 7 days, and then germinated seedlings with residual endosperm removed were transferred to plastic pot containing 1-L nutrient solution. Three replicates of three plants each pot were set for each wheat line. Pots were randomly placed and rearranged every week. All plants were grown under natural conditions with all nutrients supplied sufficiently (see below). The average lowest and highest temperatures were 7.2 and $19.9{ }^{\circ} \mathrm{C}$, respectively, and the average relative humidity was $36 \%$ during the hydroponic trial. The nutrient solution contained $(\mathrm{mM}) \mathrm{Ca}\left(\mathrm{NO}_{3}\right)_{2} 1, \mathrm{KH}_{2} \mathrm{PO}_{4} 0.2, \mathrm{MgSO}_{4}$ $0.5, \mathrm{KCl} 1.5, \mathrm{CaCl}_{2} \quad 1.5, \mathrm{H}_{3} \mathrm{BO}_{3} \quad 1 \times 10^{-3}$, $\left(\mathrm{NH}_{4}\right)_{6} \mathrm{Mo}_{7} \mathrm{O}_{24} 5 \times 10^{-5}, \mathrm{CuSO}_{4} 5 \times 10^{-4}, \mathrm{ZnSO}_{4}$
$1 \times 10^{-3}, \mathrm{MnSO}_{4} 1 \times 10^{-3}, \mathrm{Fe}(\mathrm{III})$-EDTA $1 \times 10^{-1}$. Nutrient solution was refreshed every 2 days, and the solution $\mathrm{pH}$ was adjusted to 6.0 using dilute $\mathrm{KOH}$ and $\mathrm{HCl}$ before refreshing. Plants were harvested 4 weeks after transplanting, root and shoot tissues were collected separately. Shoot dry weight (SDW), root dry weight (RDW) and tiller number (TN) were investigated. Total $\mathrm{N}$ concentration in root and shoot tissues was measured using Kjeldahl method. NUP were calculated as the sum of $\mathrm{N}$ accumulated in root $(\mathrm{RDW} \times \operatorname{root} \mathrm{N}$ concentration) and shoot $(\mathrm{SDW} \times$ Shoot $\mathrm{N}$ concentration).

\section{Detection of QTLS}

A total of 150 DHLs were randomly selected from the original 221 DHLs to construct the genetic map. The DH population and the genetic map were constructed by Institute of Crop Science, Chinese Academy of Agricultural Sciences in Beijing (Hao et al., 2003). The genetic map was constructed according to the located Xgwm primers using software Mapmaker/EXP3.0 (Hao et al., 2003, and references therein). The new version of the linkage map (which was used in this study) totally contained 395 marker loci including 132 AFLP, 112 Xgwm (Röder et al., 1998), 97 WMC (International Wheat Microsatellite Consortium), nine Xgdm (Pestsova et al., 2000), six Xpsp (developed by John Inners Centre, UK), 33 CWM and six EST loci (developed by Institute of Crop Science, Chinese Academy of Agricultural Sciences, Beijing, China), and covered $3904 \mathrm{cM}$ with $9.9 \mathrm{cM}$ per marker. The 395 loci distributed on 30 linkage groups belong to 21 chromosomes with 161, 177, and 57 loci on chromosomes of genomes A, B and D, respectively (Ruilian Jing et al., unpublished data).

QTL detection was conducted by composite interval mapping (Zeng, 1994). Analyses of QTL location, origin of positive alleles, and additive values of QTLs were performed using QTL Cartographer version 2.0 (Model 6, Basten et al., 2001). Forward regression was analyzed using a window size of $10 \mathrm{cM}$, a walk speed of $1 \mathrm{cM}$ and five control markers. The threshold for QTL detection was fixed at a LOD value of 2.0. Variance analysis and correlation coefficients between traits were analyzed using SPSS11.0 for Windows. 


\section{Results}

\section{Evaluation of phenotypes}

\section{Field trials}

In both trials, there were significant differences for NUP among the tested lines (120 DHLs and two parents) and between the two $\mathrm{N}$ treatments, and significant interactions between genotype and $\mathrm{N}$ level (Table 1). NUP was higher for the female parent Hanxuan 10 than for the male parent Lumai 14, and segregated continuously in the DH population under both $\mathrm{LN}$ and $\mathrm{HN}$ conditions in both trials (Table 1, Figure 1). LN substantially reduced NUP of the population, average NUP of the DHLs under LN condition was about $76 \%$ and $67 \%$ of that under HN condition in trials 1 and 2 , respectively. The reducing effect of LN on NUP differed among the DHLs in both trials. For example, in trial 2 relative NUP (LN/HN\%) varied from $42 \%$ to $114 \%$ (data not shown). Lower NUP under LN condition greatly reduced wheat growth in both field trials. For example in trial 2, the plant height of Hanxuan 10 was reduced from $110 \mathrm{~cm}$ under $\mathrm{HN}$ condition to $104 \mathrm{~cm}$ under LN condition, and the plant height of Lumai 14 was reduced from $75 \mathrm{~cm}$ under $\mathrm{HN}$ condition to $71 \mathrm{~cm}$ under LN condition. The grain yield of Hanxuan 10 was reduced from $6.5 \mathrm{~g} \mathrm{plant}^{-1}$ under $\mathrm{HN}$ condition to $5.3 \mathrm{~g} \mathrm{plant}^{-1}$ under $\mathrm{LN}$ condition, and the grain yield of Lumai 14 was reduced from $4.4 \mathrm{~g} \mathrm{plant}^{-1}$ under $\mathrm{HN}$ condition to $2.5 \mathrm{~g} \mathrm{plant}^{-1}$ under LN condition. The average plant height of the population was reduced from $87 \mathrm{~cm}$ (ranged from 60 to $117 \mathrm{~cm}$ ) under $\mathrm{HN}$ condition to $82 \mathrm{~cm}$ (ranged from 60 to $101 \mathrm{~cm}$ ) under LN condition, and the average grain yield of the population was reduced from $4.6 \mathrm{~g} \mathrm{plant}^{-1}$ (ranged from 1.0 to $7.3 \mathrm{~g} \mathrm{plant}^{-1}$ ) under $\mathrm{HN}$ condition to $3.3 \mathrm{~g} \mathrm{plant}^{-1}$ (ranged from 1.0 to $5.2 \mathrm{~g} \mathrm{plant}^{-1}$ ) under LN condition (data not published).

Average NUP of the population under either $\mathrm{LN}$ or $\mathrm{HN}$ condition in trial 1 was higher than the corresponding values in trial 2 (Table 1), indicating NUP varied between trials and was susceptible to environmental influence. NUP variations between the two field trials were also reflected from the moderate correlation coefficients for NUP between trials. The correlation coefficients for NUP between trials 1 and 2 were $0.656(P<0.001)$ under LN and $0.618(P<0.001)$ under HN. Significantly positive correlations for NUP between $\mathrm{N}$ levels were observed, the correlation coefficients for NUP between $\mathrm{LN}$ and $\mathrm{HN}$ were $0.752(P<0.001)$ in trial 1 and 0.690 $(P<0.001)$ in trial 2.

NUP was more closely correlated with biological and grain yields than UTE under both $\mathrm{N}$ levels (Table 2). The correlation coefficients between NUP and biological yield varied from

Table 1. Values of the traits investigated in the field and hydroponic trials. NUP $=\mathrm{N}$ uptake $\left(\mathrm{mg} \mathrm{plant}^{-1}\right), \mathrm{SDW}=\mathrm{shoot}_{\mathrm{dry}}$ weight $\left(\mathrm{mg} \mathrm{plant}^{-1}\right), \mathrm{RDW}=$ root dry weight $\left(\mathrm{mg} \mathrm{plant}^{-1}\right), \mathrm{TN}=$ tiller numbers per plant $\left(\right.$ tillers plant $\left.{ }^{-1}\right) . \mathrm{LN}=$ low $\mathrm{N}$ treatment, $\mathrm{HN}=$ high $\mathrm{N}$ treatment

\begin{tabular}{|c|c|c|c|c|c|c|c|c|c|}
\hline & \multirow{4}{*}{$\begin{array}{l}\text { Trial } \\
\text { Trait } \\
\text { Treatment }\end{array}$} & \multirow{2}{*}{\multicolumn{4}{|c|}{$\frac{\text { Field Trial }}{\text { NUP }}$}} & \multicolumn{4}{|c|}{ Hydroponic trial } \\
\hline & & & & & & \multirow[t]{3}{*}{ SDW } & \multirow[t]{3}{*}{ RDW } & \multirow[t]{3}{*}{$\mathrm{TN}$} & \multirow[t]{3}{*}{ NUP } \\
\hline & & \multicolumn{2}{|c|}{ Trial 1} & \multicolumn{2}{|c|}{ Trial 2} & & & & \\
\hline & & $\mathrm{LN}$ & $\mathrm{HN}$ & $\mathrm{LN}$ & $\mathrm{HN}$ & & & & \\
\hline \multirow[t]{2}{*}{ Parents } & Hanxuan 10 & 146 & 190 & 136 & 185 & 344 & 121 & 6.7 & 16.2 \\
\hline & Lumai 14 & 103 & 102 & 59 & 108 & 169 & 83 & 4.0 & 7.8 \\
\hline \multirow[t]{4}{*}{ Population } & Mean & 107 & 141 & 79 & 118 & 235 & 99 & 5.6 & 11.1 \\
\hline & $\mathrm{SD}$ & 18 & 22 & 16 & 21 & 52 & 12 & 1.3 & 2.4 \\
\hline & Min. & 70 & 93 & 39 & 62 & 133 & 65 & 3.4 & 6.4 \\
\hline & Max. & 155 & 214 & 115 & 172 & 357 & 134 & 8.7 & 16.2 \\
\hline \multirow[t]{3}{*}{ Analysis of variance } & Genotype & $* * *$ & & $* * *$ & & $* * *$ & $* * *$ & $* * *$ & $* * *$ \\
\hline & $\mathrm{N}$ level & $* * *$ & & $* * *$ & & & & & \\
\hline & Interaction & $* * *$ & & $* *$ & & & & & \\
\hline
\end{tabular}



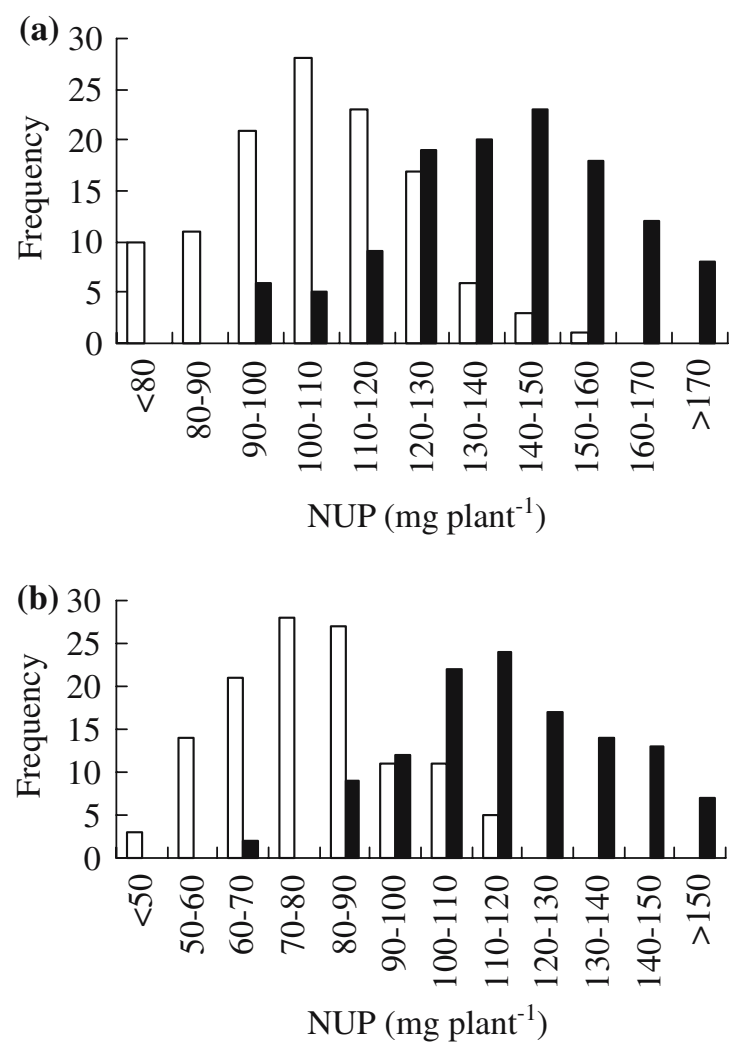

Figure 1. Frequency distribution of 120 DHLs for NUP under LN (open bar) and HN (close bar) conditions in field trials 1 (a) and 2 (b).

0.887 to 0.953 , and those between NUP and grain yield ranged from 0.755 to 0.887 . No significant positive correlation between biological yield and UTE based on biological yield was observed, but there were significant positive correlations between grain yield and UTE based on grain yield in both treatments in both trials.

\section{Hydroponic culture}

To analyze factors affecting $\mathrm{N}$ uptake, a hydroponic culture was conducted to investigate SDW, RDW, TN and NUP at seedling stage. When all nutrients were supplied sufficiently, Hanxuan 10 had much higher SDW, RDW, TN and NUP than Lumai 14 (Table 1). The DHLs also varied greatly in these four traits (Table 1). Correlation analysis showed that these four traits were highly inter-correlated (Table 3), and were all significantly and positively correlated with NUP investigated in the field trials (Table 4). In general, the correlation coefficients between TN in hydroponic culture and NUP in field trials were relatively higher, and those between RDW in hydroponic culture and NUP in field trials were relatively lower.

\section{Detection of QTLS}

\section{Field trials}

Totally 17 QTLs were detected for NUP in the two field trials (Table 5). Of the 17 NUP QTLs, nine and eight were detected under $\mathrm{LN}$ and $\mathrm{HN}$ conditions, respectively; eight were contributed by Hanxuan 10, and nine by Lumai 14 (Table 5). NUP variation explained by individual QTL varied from $5.2 \%$ to $21.9 \%$ under $\mathrm{LN}$ condition, and $6.4-15.9 \%$ under HN condition (Table 5).

The 17 NUP QTLs distributed at 13 loci on 12 chromosomes, with the chromosomes of B genome having the highest number (9) of QTLs

Table 2. Correlation coefficients between NUP, utilization efficiency and yields in the field trials. Utilization efficiency is defined as the biological or grain yield produced by unit $\mathrm{N}$ uptake

\begin{tabular}{|c|c|c|c|c|}
\hline & \multicolumn{2}{|l|}{ Trial 1} & \multicolumn{2}{|l|}{ Trial 2} \\
\hline & LN & $\mathrm{HN}$ & LN & $\mathrm{HN}$ \\
\hline & \multicolumn{4}{|l|}{ NUP } \\
\hline Biological yield & $0.887 * * *$ & $0.913 * * *$ & $0.953 * * *$ & $0.916^{* * *}$ \\
\hline \multirow[t]{2}{*}{ Grain yield } & $0.755^{* * *}$ & $0.822 * * *$ & $0.887 * * *$ & $0.856^{* * *}$ \\
\hline & \multicolumn{4}{|c|}{ Utilization efficiency based on biological yield ${ }^{\mathrm{a}}$} \\
\hline \multirow[t]{2}{*}{ Biological yield } & $0.019 * *$ & $0.060 * *$ & $-0.215^{*}$ & $0.012 * *$ \\
\hline & \multicolumn{4}{|c|}{ Utilization efficiency based on grain yield ${ }^{\mathrm{b}}$} \\
\hline Grain yield & $0.392 * * *$ & $0.403 * * *$ & $0.276^{* * *}$ & $0.486 * * *$ \\
\hline
\end{tabular}

$*, * *$, and $* * *$ indicate significant at $0.05,0.01$, and 0.001 level, respectively.

${ }^{a}$ Utilization efficiency based on biological yield was calculated as the biological yield produced by unit NUP.

${ }^{\mathrm{b}}$ Utilization efficiency based on grain yield was calculated as the grain yield produced by unit NUP. 
Table 3. Correlation coefficients between traits in the hydroponic culture. All the correlation coefficients were significant at 0.001 level

\begin{tabular}{llll}
\hline & RDW & TN & NUP \\
\hline SDW & 0.687 & 0.570 & 0.965 \\
RDW & & 0.475 & 0.675 \\
TN & & & 0.673 \\
\hline
\end{tabular}

Table 4. Correlation coefficients between NUP in the field trials and the traits investigated in the hydroponic culture. All the correlation coefficients were significant at 0.01 level

\begin{tabular}{lllllll}
\hline & & \multicolumn{5}{c}{ Hydroponic culture } \\
\cline { 4 - 7 } Field trial & & & SDW & RDW & TN & NUP \\
\hline \multirow{2}{*}{ NUP } & \multirow{2}{*}{ Trial 1 } & LN & 0.456 & 0.396 & 0.563 & 0.493 \\
& & HN & 0.338 & 0.298 & 0.387 & 0.371 \\
& \multirow{2}{*}{ Trial 2 } & LN & 0.367 & 0.333 & 0.490 & 0.407 \\
& & HN & 0.370 & 0.358 & 0.365 & 0.392 \\
\hline
\end{tabular}

(Figure 2). Four loci were detected to control NUP in different field trials. Two loci (the locus centered at Xgwm539 on chromosome 2D and the locus between Xgwm495 and Xgwm149 on
Figure 2. QTLs for investigated traits in the two field trials and the hydroponic culture. QTLs were shown as 'arrow + treatment name + trial name'. The arrows below the trait names point to the LOD peaks of QTLs. Letters and numbers above the traits indicate treatments and trials. LN and $\mathrm{HN}$ indicate low $\mathrm{N}$ and high $\mathrm{N}$ treatments in the field trials, respectively; 1 and 2 indicate field trials 1 and 2, respectively; $\mathrm{H}$ indicates hydroponic culture. QTLs with Hanxuan 10 and Lumai 14 alleles are listed on the left and the right of the chromosomes, respectively.

chromosome 4B) were found to control NUP under $\mathrm{LN}$ condition in field trial 1 and under $\mathrm{HN}$ condition in field trial 2; one locus (centered at Xgwm108 on chromosome 3B) was detected to control NUP under HN condition in both field trials; and another locus (between WMC179.1 and WMC256 on chromosome 6A) was detected to control NUP under LN condition in both field trials (Figure 2).

\section{Hydroponic culture}

For the four traits investigated in the hydroponic culture, totally 17 QTLs were detected (Table 6). For SDW, four QTLs were detected and mapped on chromosomes 1B, 2B, 5D and 7B. These loci

Table 5. QTLs for NUP (mg plant ${ }^{-1}$ ) detected in field trials

\begin{tabular}{|c|c|c|c|c|c|c|}
\hline Treatment & Trial & $\mathrm{Chr}^{\mathrm{a}}$ & Marker interval $^{\mathrm{b}}$ & LOD & $\operatorname{VE}(\%)^{\mathrm{c}}$ & $\mathrm{ADD}^{\mathrm{C}}$ \\
\hline \multirow[t]{9}{*}{$\mathrm{LN}$} & \multirow[t]{4}{*}{1} & $2 \mathrm{D}$ & Xgwm539-P4233-175 & 2.08 & 6.0 & -5 \\
\hline & & $4 B$ & Xgwm495-Xgwm149 & 2.26 & 5.2 & 4 \\
\hline & & $6 \mathrm{~A}$ & WMC179.1-WMC256 & 7.25 & 21.9 & 9 \\
\hline & & $6 \mathrm{~B}$ & $\overline{\mathrm{P} 3454-165-\mathrm{P} 3516-205}$ & 4.26 & 10.9 & -6 \\
\hline & \multirow[t]{5}{*}{2} & $2 \mathrm{~B}$ & WMC272-X $\overline{\mathrm{Xgwm} 319}$ & 3.04 & 8.3 & -5 \\
\hline & & $4 \mathrm{~A}$ & WMC89-WMC420 & 2.14 & 6.3 & 4 \\
\hline & & $5 \mathrm{~B}$ & WMC363-WMC376 & 4.14 & 12.4 & 6 \\
\hline & & $6 \mathrm{~A}$ & WMC179.1-WMC256 & 2.62 & 8.3 & 5 \\
\hline & & $7 \mathrm{D}$ & Xgdm88-WMC463 & 2.69 & 10.1 & 6 \\
\hline \multirow[t]{8}{*}{$\mathrm{HN}$} & \multirow[t]{4}{*}{1} & $3 \mathrm{~B}$ & $\overline{\mathrm{P} 2076-147-X g w m 108}$ & 2.57 & 7.0 & -6 \\
\hline & & $5 \mathrm{~A}$ & Xgwm415-Xgwm304 & 3.30 & 8.6 & -7 \\
\hline & & $5 \mathrm{~A}$ & Xgwm595-WMC410 & 5.74 & 15.9 & 9 \\
\hline & & $7 \mathrm{~B}$ & Xgwm400-P6401-238 & 2.33 & 6.8 & 6 \\
\hline & \multirow[t]{4}{*}{2} & $1 \mathrm{~B}$ & WMC156-P3446-183 & 2.40 & 6.4 & -6 \\
\hline & & $2 \mathrm{D}$ & Xgwm157-Xgwm539 & 4.31 & 14.0 & -9 \\
\hline & & $3 \mathrm{~B}$ & Xgwm108-WMC291 & 2.67 & 7.3 & -7 \\
\hline & & $4 B$ & Xgwm495-Xgwm149 & 4.24 & 14.1 & 9 \\
\hline
\end{tabular}

${ }^{\mathrm{a}} \mathrm{Chr}$ means chromosome name.

${ }^{\mathrm{b}}$ Markers underlined were the nearest marker to the QTL.

${ }^{c} \mathrm{VE}(\%)$ means variation explained.

${ }^{\mathrm{d}} \mathrm{ADD}$ is abbreviation of additive effects, a negative sign means that the positive alleles come from the parent Hanxuan 10 , while a positive sign means positive alleles come from the parent Lumai 14. 

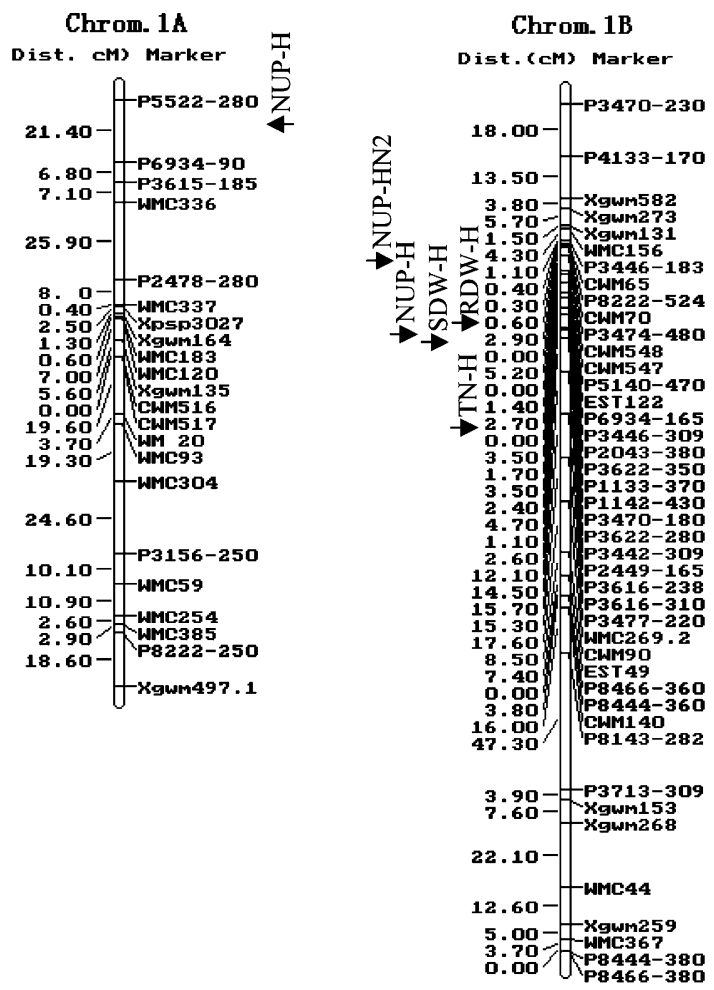

Chrom. 3A

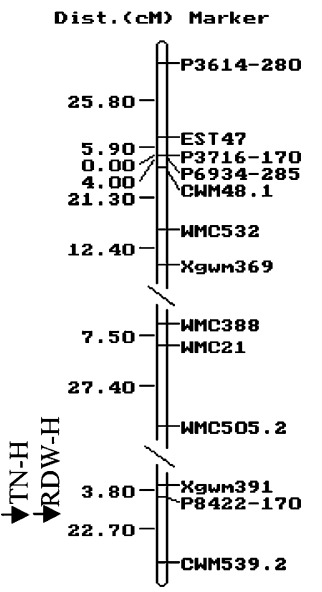

Dist. (cM) Marker
Chrom. 2B

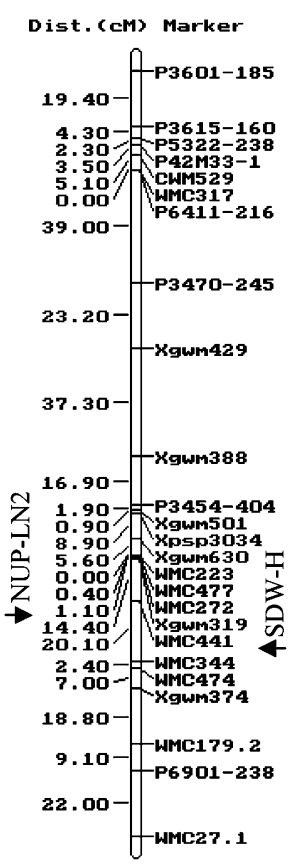

Chrom. 3D

Dist. (cM) Marker

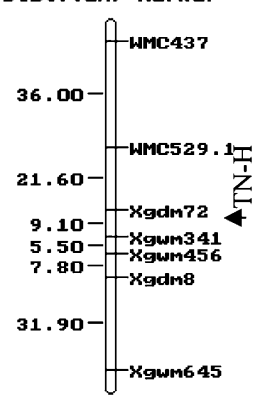

Chrom. 2D

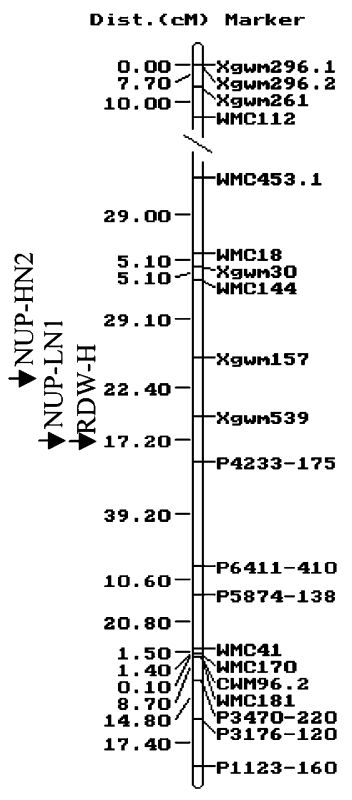

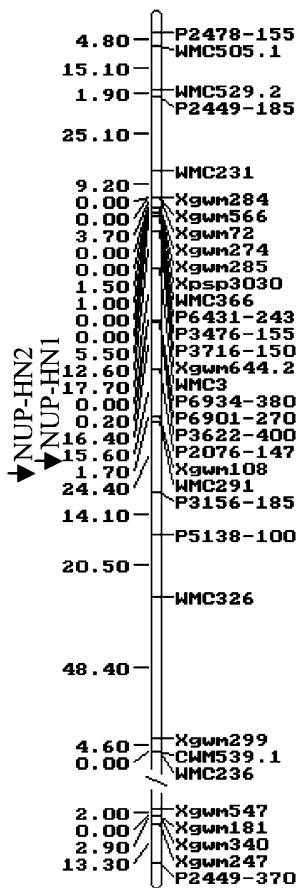

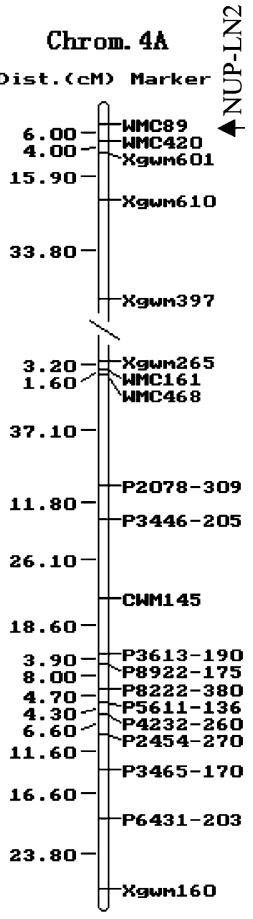


Chrom. 4B

Dist.(cM) Marker

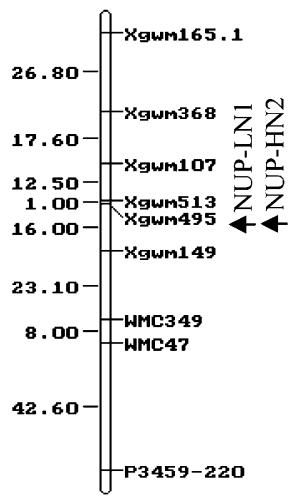

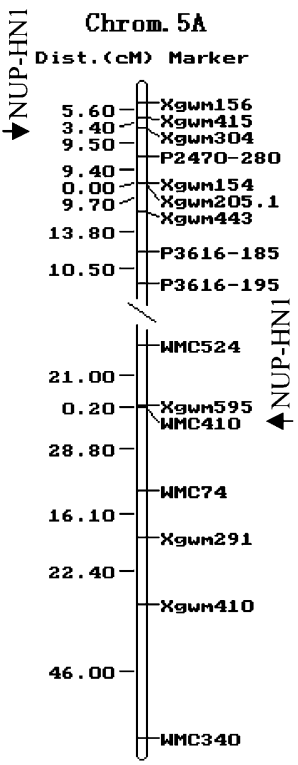

Chrom. 7A

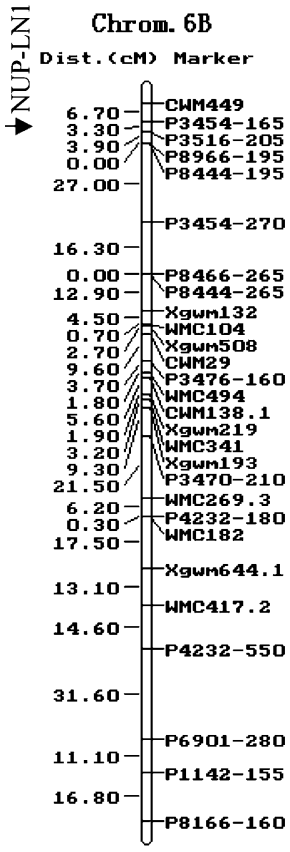

Dist. (cM) Marker

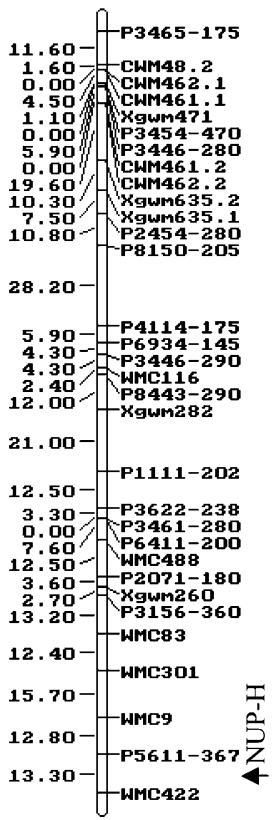

Chrom. 5B

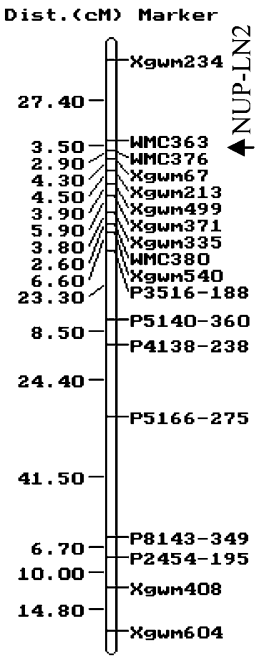

Chrom. 5D
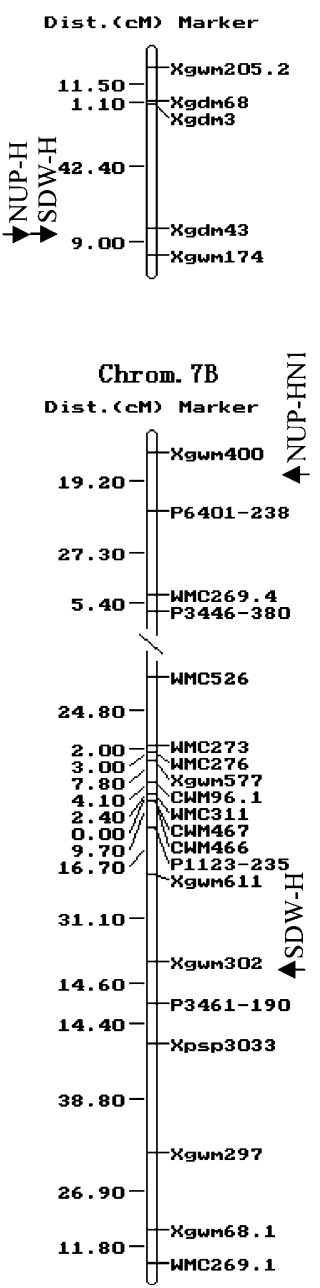

Chrom. 6A

Dist. (cM) Marker

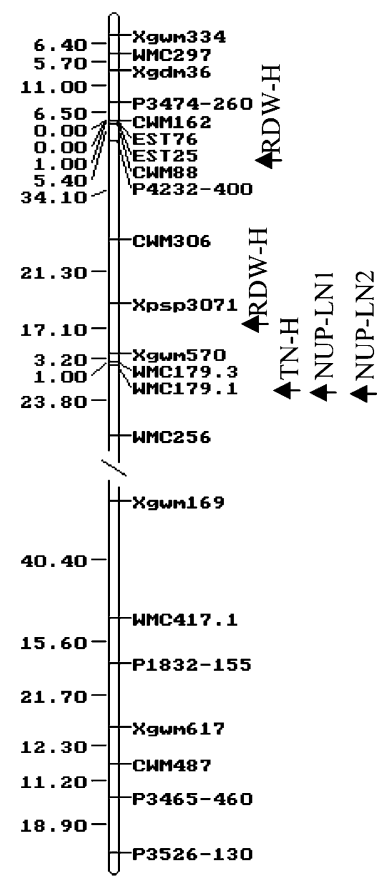

Chrom. 7D

Dist. (cM) Marker

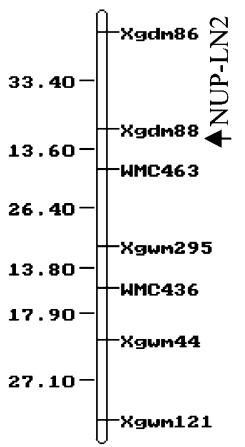

NUP $=\mathrm{N}$ uptake

SDW $=$ Shoot dry weight

RDW = Root dry weight

$\mathrm{TN}=$ Tiller number per plant

Figure 2. Continued. 
Table 6. QTLs detected in the hydroponic culture

\begin{tabular}{|c|c|c|c|c|c|}
\hline Trait & $\mathrm{Chr}^{\mathrm{a}}$ & Marker interval $^{\mathrm{b}}$ & LOD & $\operatorname{VE}(\%)^{\mathrm{c}}$ & $\mathrm{ADD}^{\mathrm{d}}$ \\
\hline \multirow[t]{4}{*}{ SDW (mg plant ${ }^{-1}$ ) } & $1 \mathrm{~B}$ & P3474-480-CWM548 & 4.36 & 10.0 & -18 \\
\hline & $2 \mathrm{~B}$ & WMC441-WMC344 & 3.70 & 8.4 & 17 \\
\hline & $5 \mathrm{D}$ & Xgdm43-Xgwm174 & 4.21 & 10.7 & -18 \\
\hline & $7 \mathrm{~B}$ & Xgwm302-P3461-190 & 2.15 & 7.9 & 15 \\
\hline \multirow[t]{5}{*}{ Root DW (mg plant ${ }^{-1}$ ) } & $1 \mathrm{~B}$ & $\overline{\text { CWM70-P3474-480 }}$ & 6.86 & 19.6 & -5 \\
\hline & $2 \mathrm{D}$ & Xgwm539-P4233-175 & 2.65 & 11.0 & -4 \\
\hline & $3 \mathrm{~A}$ & P8422-170-CWM539.2 & 2.81 & 10.4 & -4 \\
\hline & $6 \mathrm{~A}$ & $\overline{\text { EST25-CWM }} 88$ & 4.02 & 11.0 & 4 \\
\hline & $6 \mathrm{~A}$ & $\overline{\mathrm{Xpsp3}} 071-\mathrm{Xgwm} 570$ & 2.37 & 8.6 & 4 \\
\hline \multirow[t]{4}{*}{ TN (tillers plant ${ }^{-1}$ ) } & 1B & P6934-165-P3446-309 & 4.38 & 11.0 & -0.4 \\
\hline & $3 \mathrm{~A}$ & P8422-170-CWM539.2 & 2.07 & 6.7 & -0.3 \\
\hline & $3 \mathrm{D}$ & Xgdm72-Xgwm341 & 4.36 & 12.5 & -0.5 \\
\hline & $6 \mathrm{~A}$ & WMC179.1-WMC256 & 4.69 & 12.9 & 0.5 \\
\hline \multirow[t]{4}{*}{ NUPn (mg plant ${ }^{-1}$ ) } & $1 \mathrm{~A}$ & $\overline{\mathrm{P} 5522-280-}$-P6934-90 & 2.42 & 6.2 & 0.6 \\
\hline & $1 \mathrm{~B}$ & $\overline{\mathrm{P} 3474-480-C W M 548}$ & 4.50 & 10.1 & -0.8 \\
\hline & $5 \mathrm{D}$ & 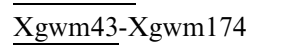 & 4.91 & 12.6 & -0.9 \\
\hline & $7 \mathrm{~A}$ & P5611-367-WMC422 & 2.07 & 4.3 & 0.5 \\
\hline
\end{tabular}

${ }^{\mathrm{a}} \mathrm{Chr}$ means chromosome name.

${ }^{\mathrm{b}}$ Markers underlined were the nearest marker to the QTL.

${ }^{\mathrm{c}} \mathrm{VE}(\%)$ means variation explained.

${ }^{\mathrm{d}} \mathrm{ADD}$ is abbreviation of additive effects, a negative sign means that the positive alleles come from the parent Hanxuan 10, while a positive sign means positive alleles come from the parent Lumai 14.

could explain $7.9-10.7 \%$ of SDW variation individually. Five QTLs, located on chromosomes $1 \mathrm{~B}, 2 \mathrm{D}, 3 \mathrm{~A}$ and $6 \mathrm{~A}$, were found to control RDW. The most significant QTL for RDW was located on chromosome $1 \mathrm{~B}$ and could explain $19.6 \%$ of RDW variation in the $\mathrm{DH}$ population. Four QTLs were detected to control TN. These TN QTLs were mapped on 1B, 3A, 3B and 6A, and could explain $6.7-12.9 \%$ of $\mathrm{TN}$ variation. For NUP four QTLs on chromosomes 1A, 1B, $5 \mathrm{~A}$ and $7 \mathrm{D}$ were detected. Phenotypic variation of NUP explained by individual NUP QTL varied from $4.3 \%$ to $12.6 \%$.

\section{Discussion}

NUP and UTE are the two components of NUE. An ideal $\mathrm{N}$-use efficient wheat variety would acquire $\mathrm{N}$ from soil efficiently, and use the absorbed $\mathrm{N}$ efficiently to produce biomass or grain yield. In present study, when a set of DHLs derived from Hanxuan 10 and Lumai 14 were grown under $\mathrm{LN}$ and $\mathrm{HN}$ conditions in two consecutive fields trials, NUP was found to be more closely correlated with biological and grain yields than UTE under both $\mathrm{N}$ levels (Table 2), confirming the previously reported results in wheat (Dhugga and Waines, 1989; Van Sanford and Mackown, 1986). These results indicated the importance of understanding the genetic control of NUP in breeding wheat with improved NUE. Therefore, in this study efforts were made to investigate the genetic control of NUP and factors affecting, NUP.

Although the two parents were originally chosen because of large difference in drought tolerance (Hanxuan 10 is drought tolerant and Lumai 14 is drought sensitive) (Hao et al., 2003), in present study large differences between the two parents and among their progenies were observed for NUP at physiological maturity stage under both LN and HN conditions in the field trials, and for SDW, RDW, TN and NUP at seedling stage in the hydroponic culture (Table 1). In the two field trials, female parent Hanxuan 10 had higher NUP than male parent Lumai 14 under both $\mathrm{N}$ levels (Table 1). Their progenies also differed greatly in NUP under either LN or HN condition (Table 1), and NUP segregated continuously in the population (Figure 1). The facts that the significant and positive correlation 
coefficients ( $r=0.656$ under LN and $r=0.618$ under HN) for NUP between trials 1 and 2 and the lower NUP of the population in trial 2 than in trial 1 (Table 1) reflected that NUP was under genetic control and also susceptible to environmental influence. Although variance analysis indicated that there was no significant interaction between trial and genotype under either $\mathrm{LN}$ or $\mathrm{HN}$ condition, there was significant difference between trials under either $\mathrm{LN}$ or $\mathrm{HN}$ condition (data not shown). The lower NUP in trial 2 was likely due to environmental influence, as trial 2 had a relative colder season before stem elongation. Another possible reason for the lower NUP in trial 2 might be the lower soil nitrate level in trial 2, for the average nitrate concentration in $0-75 \mathrm{~cm}$ soil profile sampled before fertilization treatment was $15 \mathrm{mg} \mathrm{N} \mathrm{kg}^{-1}$ soil in trial 1 and $6 \mathrm{mg} \mathrm{N} \mathrm{kg}^{-1}$ in trial 2.

In order to analyze factors affecting NUP, SDW, RDW, TN and NUP were investigated in a hydroponic culture with $2 \mathrm{mM}$ nitrate and other essential nutrients in the solution. All the four traits showed significant differences between the two parents and among the DHLs (Table 1), and segregated continuously in the population in the hydroponic culture. All the four traits were significantly and positively correlated with each other; and the closest correlation was between NUP and SDW (Table 3), indicating that vigorous DHLs generally had higher NUP. The much lower NUP in the hydroponic culture than in the field trials (Table 1) was mainly because the hydroponic culture was harvested much earlier than the field trials, and might not be caused by the low nitrate concentration supplied to the plants. In this hydroponic culture a nitrate concentration of $2 \mathrm{mM}$ was chosen because of the fact it was able to meet the $\mathrm{N}$ requirement of wheat seedlings under our experimental conditions in a preliminary experiment, and it might prevent the inhibition of high $\mathrm{N}$ concentration on root development.

This study totally detected 21 QTLs for NUP of which 17 were detected in the two field trials, and four were detected in the hydroponic culture (Tables 5 and 6). The 21 NUP QTLs distributed at 16 loci on 14 chromosomes (Figure 2). Of the 16 loci contributed to NUP, five loci were detected in more than one trial (Figure 2). The first locus (between WMC156 and CWM148 on chromosome 1B) contained a minor QTL for NUP under HN condition in field trial 2 and a minor QTL for NUP in the hydroponic culture (Tables 5 and 6, Figure 2). QTLs for SDW, RDW and $\mathrm{TN}$ in the hydroponic culture were also mapped around this locus (Figure 2). The second locus (centered at Xgwm539 on chromosome 2D) contained a QTL for NUP under LN condition in field trial 1 and a QTL for NUP under $\mathrm{HN}$ condition in field trial 2 (Figure 2). This locus is also associated with a QTL for RDW in the hydroponic culture. In the comparable regions of these two loci (on chromosomes $1 \mathrm{~B}$ and 2D), QTLs for tiller number per square meter have been reported (Huang et al., 2003). It seems that these two loci may confer genes controlling tiller number. As wheat root systems develop in a coordinated pattern along with tillers (Weaver, 1926), plants with more tillers may develop more roots, and thus can absorb $\mathrm{N}$ more efficiently from soil. The third locus (centered at Xgwm108 on chromosome 3B) contained two minor QTLs for NUP under HN condition in the two field trials (Table 5, Figure 2). The fourth locus (between Xgwm495 and Xgwm149 near the centromere of chromosome 4B) contained a QTL for NUP under LN condition in field trial 1 and a QTL for NUP under HN condition in field trial 2 (Figure 2). Around the centromere of chromosome 4B, QTLs for ears per plant and grain number per ear under $\mathrm{N}$ stress condition and a major QTL for grain number per ear under normal condition were detected using a spring wheat $\mathrm{DH}$ population (Quarrie et al., 2005). These results indicate the centromere region of chromosome 4B is possibly involved in the control of wheat performance under both $\mathrm{LN}$ and $\mathrm{HN}$ conditions. Finally, the fifth locus (between WMC179.1 and WMC256 on chromosome 6A) was detected to control NUP under LN in both field trials (Figure 2); the NUP QTL under LN condition in field trial 1was the most significant QTL detected in this study, and could explain $21.9 \%$ of phenotypic variation (Table 5). The consistent expression of this locus under LN suggests that it is an important locus in controlling NUP under LN condition. The control of this locus in NUP under LN condition was possibly due to its control of RDW, for around this locus a QTL for RDW was detected in the hydroponic culture (Figure 2). In the comparable region of this locus on 
chromosome 6A, a major QTL for grain number per ear was detected under $\mathrm{N}$ stress condition (Quarrie et al., 2005), further confirming the importance of this locus under $\mathrm{N}$ stress condition. The linkages between the QTLs for NUP from different trials and from different $\mathrm{N}$ treatments at above five loci reflected the positive correlations for NUP between trials and between $\mathrm{N}$ levels. However, under either LN or HN condition only one locus (the locus on chromosome 6A under $\mathrm{LN}$ condition and the locus on chromosome $3 \mathrm{~B}$ under HN condition) was repeatedly detected to control NUP in the field trials, these was possibly due to the variation of NUP between trials that has been discussed above. The low reproducibility of NUP QTLs indicated more research is needed to detect NUP QTLs.

Greater early vigor has been suggested as a useful trait for yield improvement of wheat crops under drought stress condition (Rebetzke and Richards, 1999; Richards et al., 2002). Early vigorous growth has also been suggested as a major factor influencing $\mathrm{N}$ uptake in wheat (Liao et al., 2004). The higher $\mathrm{N}$ uptake of vigorous wheat genotype has been associated with vigorous early root and shoot growth (Liao et al., 2004). In the hydroponic culture of this study, the drought-tolerant parent Hanxuan 10 also had greater early vigor (higher SDW, RDW and TN) and higher NUP than the drought-sensitive parent Lumai 14 (Table 1). The close correlation between NUP and SDW in the population also suggested that vigorous DHLs generally had high NUP (Table 4). The four traits were significantly and positively correlated with NUP measured under both LN and HN conditions in both field trials (Table 4). Consequently, linkages between the NUP QTLs in the field trials and the QTLs for SDW, RDW and TN in the hydroponic culture were observed (Figure 2). Around three (on chromosomes 1B, 2D and 6A) of the five NUP loci discussed above, QTLs for RDW were detected (Figure 2). One (on chromosome 1B and 5D) of the four SDW QTLs and two (on chromosomes $1 \mathrm{~B}$ and 6A) of the four TN QTLs were linked with QTLs for NUP in the field trials (Figure 2). Although the locus (controlling NUP under LN condition in trial 1 and $\mathrm{HN}$ condition in trial 2) between markers Xgwm495 and Xgwm149 on chromosome 4B was not detected to control early growth of wheat plant in this work (Figure 2), around this region a major QTL governing coleoptile length has been reported, and the same QTL affects leaf size, coleoptile tiller size and overall seedling vigor (Rebetzke et al., 2001). Considering that the removal of the residual endosperm from germinating seeds in the hydroponic culture may affect the QTLs established in hydroponic culture compared to the field trials, linkage between the NUP QTLs and QTLs for SDW and TN of wheat seedlings grown on high fertility soils in two independent pot trials was analyzed. Around or at the three loci (on chromosomes 1B, 2D and 6A) of the five loci that were repeatedly detected to control NUP in this study (Figure 2), QTLs for TN or SDW were also detected in the pot trials. Moreover, SDW and $\mathrm{TN}$ in the pot trials were significantly and positively correlated with NUP in the field trials (Tong et al., unpublished data). Therefore, the present study provided both physiological and genetic evidence to support the conclusion that increased seedling growth has a positive influence on NUP at maturity stage under both LN and HN conditions.

In summary, the present study demonstrated that $\mathrm{N}$ uptake by wheat under either low $\mathrm{N}$ stress or normal condition was controlled by polygenes. The fact that some loci were repeatedly detected to control $\mathrm{N}$ uptake under $\mathrm{LN}$ or $\mathrm{HN}$ condition implied that $\mathrm{N}$ uptake could be improved through molecular breeding from wide range of soil $\mathrm{N}$ scenarios. The positive influence of early vigor on $\mathrm{N}$ uptake may offer valuable clues for further research on genetic mechanisms of $\mathrm{N}$ uptake, and facilitate wheat breeding with increased $\mathrm{N}$ uptake efficiency.

\section{Acknowledgements}

This research was supported by grants (30390083, 30521001， 2004CB117200, 2005CB120904, 2003AA207080, KSCX2-SW-304 and 05-02-01A) from the National Natural Sciences foundation of China, the Ministry of Science and Technology of China, the Ministry of Agriculture of China and the Chinese Academy of Sciences. 


\section{References}

Agrama H A S, Zakaria A G, Said F B and Tuinstra M R 1999 Identification of quantitative trait loci for nitrogen use efficiency in maize. Mol. Breed. 5, 187-195.

Basten C J, Weir B S and Zeng Z B 2001 QTL CARTOGRAPHER, Version 1.15. Department of Statistics, North Carolina State University, Raleigh.

Bertin P and Gallais A 2000 Physiological and genetic basis of nitrogen use efficiency in maize: I. QTL detection and coincidences. Maydica 45, 67-80.

Crawford N M and Forde B G 2002 Molecular and developmental biology of inorganic nitrogen nutrition. In The Arabidopsis Book. Eds. E Meyerowitz and C Somerville. American Society of Plant Biologists, Rockville, MD, doi/ 10.1199/tab.0011, http://www.aspb.org/publications/arabidopsis.

Dhugga K S and Waines J G 1989 Analysis of nitrogen accumulation and use in bread and durum wheat. Crop Sci. 29, 1232-1239.

Gallais A and Hirel B 2004 An approach to the genetics of nitrogen use efficiency in maize. J. Exp. Bot. 55, 295-306.

Hao Z F, Chang X P, Guo X J, Jing R L, Li R Z and Jia J Z 2003 QTL mapping for drought tolerance at stage of germination and seedling in wheat (Triticum aestivum L.) using a DH population. Agric. Sci. China 2, 943-949.

Hirel B, Bertin P, Quillere I, Bourdoncle W, Attagnant C, Dellay C, Gouy A, Cadiou S, Retailliau C, Falque M and Gallais A 2001 Towards a better understanding of the genetic and physiological basis for nitrogen use efficiency in maize. Plant Physiol. 125, 1258-1270.

Huang X Q, Cöster H, Ganal M W and Röder M S 2003 Advanced backcross QTL analysis for the identification of quantitative trait loci alleles from wild relatives of wheat (Triticum aestivum L.). Theor. Appl. Genet. 106, 1379-1389.

Ishimaru K, Kobayashi N, Ono K, Yano M and Ohsugi R 2001 Are contents of Rubisco, soluble protein and nitrogen in flag leaves of rice controlled by the same genetics?. J. Exp. Bot. 52, 1827-1833.

Jing R L, Chang X P, Jia J Z and Hu R H 1999 Establishing wheat doubled haploid population for genetic mapping by anther culture. Biotechnology 9(3), 4-8.

Ju X T, Liu X J, Zhang F S and Roelcke M 2004 Nitrogen fertilization, soil nitrate accumulation, and policy recommendations in several agricultural regions of China. Ambio 33, 300-305.

Lian X M, Xing Y Z, Yan H, Xu C G, Li X H and Zhang Q F, 2005 QTLs for low nitrogen tolerance at seedling stage identified using a recombinant inbred line population derived from an elite rice hybrid. Theor. Appl. Genet. 112, 85-96.

Liao M, Fillery I R P and Palta J A 2004 Early vigorous growth is a major factor influencing nitrogen uptake in wheat. Func. Plant Biol. 31, 121-129.

Loudet $\mathrm{O}$, Chaillou S, Merigout P, Talbotec $\mathbf{J}$ and DanielVedele F 2003 Quantitative trait loci analysis of nitrogen use efficiency in Arabidopsis. Plant Physiol. 131, 345-358.

Obara M, Kajiura M, Fukuta Y, Yano M, Hayashi M, Yamaya T and Sato T 2001 Mapping of QTLs associated with cytosolic glutamine synthetase and NADH-glutamate synthase in rice (Oryza sativa L.). J. Exp. Bot. 52, 1209-1217.

Obara M, Sato T, Sasaki S, Kashiba K, Nagano A, Nakamura I, Ebitani T, Yano M and Yamaya T 2004 Identification and characterization of a QTL on chromosome 2 for cytosolic glutamine synthetase content and panicle number in rice. Theor. Appl. Genet. 110, 1-11.

Ortiz-Monasterio J I, Sayre K D, Rajaram S and McMahon M 1997 Genetic progress in wheat yield and nitrogen use efficiency under four nitrogen rates. Crop Sci. 37, 898-904.

Pestsova E, Ganal M W and Röder M S 2000 Isolation and mapping of microsatellite markers specific for the $\mathrm{D}$ genome of bread wheat. Genome 43, 689-697.

Presterl T, Seitz G, Landbeck M, Thiemt E M, Schmidt W and Geiger H H 2003 Improving nitrogen-use efficiency in European maize: estimation of quantitative genetic parameters. Crop Sci. 43, 1259-1265.

Quarrie S A, Steed A, Calestani C, Semikhodskii A, Lebreton C, Chinoy C, Steele N, Pljevljakusic D, Waterman E, Weyen J, Schondelmaier J, Habash D Z, Farmer P, Saker L, Clarkson D T, Abugalieva A, Yessimbekova M, Turuspekov Y, Abugalieva S, Tuberosa R, Sanguineti M C, Hollington P A, Aragues R, Royo A and Dodig D 2005 A high-density genetic map of hexaploid wheat (Triticum aestivum L.) from the cross Chinese Spring $\times$ SQ1 and its use to compare QTLs for grain yield across a range of environments. Theor. Appl. Genet. 110, 865-880.

Raun W R and Johnson G V 1999 Improving nitrogen use efficiency for cereal production. Agron. J. 91, 357-363.

Rebetzke G J and Richards R A 1999 Genetic improvement of early vigour in wheat. Aust. J. Agric. Res. 51, 235-245.

Rebetzke G J, Appells R, Morrison A, Richards R A, McDonald G, Ellis M H, Spielmeyer W and Bonnett D G 2001 Quantitative trait loci on chromosome 4B for coleoptile length and early vigour in wheat (Triticum aestivum L.). Aust. J. Agric. Res. 52, 1221-1234.

Richards R A, Rebetzke G J, Condon A G and van Herwaarden A F 2002 Breeding opportunities for increasing the efficiency of water use and crop yield in temperate cereals. Crop Sci. 42, 111-121.

Röder M S, Korzun V, Wendehake K, Plaschke J, Tixier M H, Leroy P and Ganal M W 1998 A microsatellite map of wheat. Genetics 149, 2007-2023.

Tilman D. 1999 Global environmental impacts of agricultural expansion: The need for sustainable and efficient practices. Proc. Natl. Acad. Sci. USA 96, 5995-6000.

Van Sanford D A and Mackown C T 1986 Variation in nitrogen efficiency among soft red winter wheat genotypes. Theor. Appl. Genet. 72, 158-163.

Van Sanford D A, Mackown C T and Cornelius P L 1991 Genetic variation for nitrogen use in soft winter wheat populations. Crop Sci. 31, 626-630.

Weaver J E 1926 Root Development of Field Crops. McGrawHill Book Company, Inc.

Yamaya T, Obara M, Nakajima H, Sasaki S, Hayakawa T and Sato T 2002 Genetic manipulation and quantitative-trait loci mapping for nitrogen recycling in rice. J. Exp. Bot. 53, 917925.

Zeng Z B 1994 Precision mapping of quantitative trait loci. Genetics 136, 1457-1468.

Zhu Z L and Chen D L 2002 Nitrogen fertilizer use in China Contributions to food production, impacts on the environment and best management strategies. Nutr. Cycl. Agroecosyst. 63, 117-127.

Section Editor: H.J. Kronzucker 